\title{
Breast cancer vaccines: maximizing peptide-based vaccines by regulatory $T$ cell depletion and toll-like receptor 9 activation Pilar Nava-Parada*1, Adam Herron ${ }^{1}$, Keith L Knutson ${ }^{1}$, Larry Pease ${ }^{1}$ and Esteban Celis ${ }^{2}$
}

\begin{abstract}
Address: ${ }^{1}$ Immunology, Mayo Clinic, Rochester, MN, USA and ${ }^{2} \mathrm{H}$. Lee Moffitt Cancer Center, University of South Florida, Tampa, FL, USA
\end{abstract}
* Corresponding author

from 24th Annual Meeting of the National Cancer Institute of Mexico

Mexico City, Mexico. 14-17 February 2007

Published: 5 February 2007

BMC Cancer 2007, 7(SuppI I):A2 doi:10.II86/I47I-2407-7-SI-A2

This article is available from: http://www.biomedcentral.com/I47I-2407/7/SI/A2

(C) 2007 Nava-Parada et al; licensee BioMed Central Ltd.

\section{Background}

Breast cancer is the most prevalent cancer in the world due to high incidence and favorable prognoses. Relapse is the major cause of mortality and will occur in up to $65 \%$ of patients with node-positive disease. Thus, effective longterm protection strategies are needed for preventing relapse. One approach that has generated interest in recent years is cancer vaccines. One of the problems with the translation of cancer vaccines is that multiple boosters are required in order to generate high-avidity memory immunity that is able to recognize naturally processed antigens. Our goal in this study was to determine if the need for boosters could be eliminated by preconditioning the immune system with anti-CD25 antibodies, which is known to deplete regulatory $\mathrm{T}$ cells, the latter of which are known to regulate the development of high-avidity memory T cells.

\section{Materials and methods}

We first developed a single peptide vaccine to generate neu-specific CTL immunity in the Neu-T mice. Neu-T mice have targeted expression of the activated rat neu in breast tissue that occurs at 3-4 weeks of age, and these mice develop spontaneous breast tumors at 15-17 weeks.

\section{Results}

The vaccine peptide, TYVPANASL (p66-74), was predicted by the SYFPEITHI algorithm http://www.syf peithi.com to bind to mouse MHC class I, H-2d and was admixed with IFA and CpG. In the non-tolerant, Balb/c mice, vaccination prevented tumor development with only a single immunization. In the tolerant neu-T mice, the same vaccine succeeded in preventing spontaneous and transplanted tumors but only when given with repeated boosters. The response was durable as it was found that $80 \%$ of mice remained tumor free by week 45 of age. In order to determine if CD25+ Tregs may be preventing or regulating the development of high avidity memory immunity, anti-CD25 monoclonal antibodies were used to deplete Tregs prior to immunization. Following depletion, a single vaccination administered at week 10 following birth, when diffuse atypical hyperplasia is already evident in the mammary glands, vaccination induced a sufficient CTL response that has kept $100 \%$ of Neu-T mice tumor free until week 31 . Immune monitoring revealed that Treg depletion resulted in higher avidity $\mathrm{T}$ cells following a single immunization.

\section{Conclusion}

Our results show that by combining a strong adjuvant (CpG) and an immunogenic peptide, we can induce an immune response capable of preventing spontaneous tumors when repetitive vaccinations are administered. Importantly, the need for boosters is reduced when Tregs are depleted prior to immunization. Removing or reducing the need for booster immunizations may facilitate the clinical translation of cancer vaccines for prevention of breast cancer development and relapse. 